\title{
PSICOLOGÍA Y PSICOLOGÍA CLÍNICA: SOBRE ÁRBOLES Y RAMAS
}

\author{
Amparo Belloch Fuster \\ Facultad de Psicología. \\ Universidad de Valencia (España).
}

\section{RESUMEN}

En su evolución conceptual y profesional la psicología clínica es deudora, sin duda, de la psicología. Pero, al mismo tiempo, su propio objeto de estudio (los trastornos mentales y del comportamiento) no siempre ha sido bien visto ni aceptado por la psicología científica, lo que ha propiciado que los psicólogos clínicos buscaran respuestas a sus preguntas, no solo en la psicología "madre", sino también en otros campos científicos diferentes. En este artículo se ofrece una visión subjetiva y por tanto, personal, de los encuentros y desencuentros entre la psicología clínica y la no-clínica (y entre los profesionales de uno y otro ámbito). Se examinan también las posibles razones que explican el éxito actual de la psicología clínica en el ámbito de los trastornos mentales y del comportamiento, las contribuciones de la psicología en general a ese éxito, y los riesgos que conlleva confiar en exceso en lo logrado hasta ahora como garantía de éxitos y logros futuros.

Palabras clave: psicología; psicología clínica; psicoterapias eficaces; diagnóstico psicológico 


\section{ABSTRACT}

Clinical Psychology has undoubtedly their roots in the psychological science. However, it must be recognized also that the study of mental disorders, the main goal for clinical psychologists, has not been always well admitted by the nonclinical psychologists. As a result, clinical psychologists have been searching for solutions to their problems in other nonpsychological scientific settings. In this paper, a subjective view about the agreements and misunderstandings between clinical and non-clinical psychology is offered. Some reasons explaining the current successes of clinical psychology are also discussed, together with the contribution of psychology to these successes and the risks involved in considering the achievements as a guarantee of future success.

\section{Key words: Psychology; clinical psychology; empirically based psychotherapies; psychological diagnosis.}

\section{INTRODUCCIÓN}

Cuando se habla de la psicología clínica se tiene la tendencia lógica y comprensible a describirla como una rama o extensión natural de la Psicología. Hablar de ramas significa que hay un tronco, una estructura viva más antigua, que sirve de sustento y proporciona identidad a las ramas. Sin embargo, desde un punto de vista histórico o fundacional, esta secuencia no es tan obvia, secuencial, o lineal como podría parecer a primera vista cuando hablamos de la psicología (tronco) y la psicología clínica (rama).

Siguiendo con la metáfora vegetal: en ocasiones sucede que una rama adquiere tal fuerza y entidad que se convierte, a su vez, en tronco de otras ramas, y puede llegar a vivir y crecer separada ya del tronco original. Este hecho lo podemos observar con frecuencia en la naturaleza: ramas que sobreviven desgajadas de su tronco inicial, otras que se convierten en troncos tanto o más potentes que el originario y adquieren con el tiempo una identidad propia tan par- 
ticular que resulta difícil saber cuál fue su "célula madre" primigenia. Pero, como no podría ser de otro modo, el proceso de generación de ramas que acaban convertidas en tronco sigue su curso, instaurándose así una secuencia que a priori es interminable y, lo que es más importante, inexorable.

Las condiciones para que este proceso de creación y re-creación se inicie y consolide son muchas y variadas: características ambientales, capacidad de adaptación y supervivencia, capacidad de buscar nuevos nichos ecológicos en los que asentarse y crecer, calidad y cantidad de los nutrientes que estén al alcance, o capacidad para buscar activamente y asimilar otros, son solamente algunas de tales condiciones. Esto no es desde luego nada nuevo ni extraño, pues como psicólogos sabemos que la historia personal de aprendizajes y experiencias, de interacción constante con el medio, es tan importante (cuando no más) que la carga genética heredada a la hora de conformar la propia identidad. $Y$ al mismo tiempo, ese proceso de configuración de la identidad no acaba sino con la desaparición física del individuo.

Este breve artículo pretende reflexionar sobre las relaciones entre psicología y psicología clínica, en primer lugar, y sobre la diversificación actual de la psicología clínica y los riesgos que ello comporta a continuación. La finalidad de estas reflexiones es encontrar algunas respuestas que permitan entender y situar en su contexto los desencuentros que se han producido, se están produciendo $y$, seguramente, se producirán, entre la psicología y la psicología clínica, tanto por lo que se refiere a la asunción de conceptos y métodos, como al ámbito de lo aplicado y profesional. Para terminar, debe aclararse que todo lo que aquí se expresa responde a la propia y subjetiva opinión personal de la autora, y por lo tanto, las referencias a otros autores en busca de otras opiniones que sustenten o avalen de algún modo las propias, serán escasas ( $\mathrm{y}$, en todo caso, su elección obedece asimismo a criterios subjetivos, como no podía ser de otro modo). 


\section{PSICOLOGÍA Y PSICOLOGÍA CLÍNICA: LOS ORÍGENES (RE)CONOCIDOS.}

En su evolución conceptual y profesional la psicología clínica es deudora, sin duda, de la psicología. Pero, al mismo tiempo, su propio objeto de estudio (los trastornos mentales y del comportamiento) no siempre ha sido bien visto ni aceptado por la psicología científica, lo que ha propiciado que los psicólogos clínicos buscaran respuestas a sus preguntas, tanto conceptuales como metodológicas, no solo en la psicología "madre", sino también en otros campos científicos diferentes, que van desde la sociología y la antropología cultural, hasta la biología y la medicina.

Una de las razones que quizá explique la muchas veces observada falta de entendimiento entre la psicología y la psicología clínica, reside en la "antigüedad científica" de sus propios objetos de estudio y, como consecuencia, de los marcos conceptuales en los que históricamente se fueron situando una y otra: mientras que el estudio de la mente y el comportamiento humanos entran en la senda de la reflexión científica en el siglo XIX, el interés por conocer los trastornos y enfermedades mentales caminó por esa senda, aunque con paso irregular y aciertos dispares, desde al menos el siglo $\mathrm{V}$ a. de $\mathrm{C}$. , es decir, desde los albores de lo que hoy conocemos como medicina.

No voy a extenderme aquí sobre los sobradamente conocidos argumentos y datos históricos que justifican y explican el nacimiento de la psicología como disciplina científica independiente allá por el último cuarto del siglo XIX. Pero quizá sea útil recordar brevemente cuándo y cómo surge la psicología, aun a costa de retomar algunos argumentos ya expresados en otros lugares por mí misma (Belloch, 1997, 2005), así como por autores reconocidos y mucho más autorizados para ello (por ej., Reisman, 1976).

La psicología, como disciplina separada de la reflexión filosófica, surge en la Alemania del último cuarto del siglo XIX, que sin duda ejercía un papel de liderazgo en todo lo relativo a la cultura y la ciencia en el mundo occidental, como consecuencia, entre otras cosas, del enorme prestigio e influencia conseguidos en su calidad de vencedora de la guerra Franco-Prusiana en 1870. Pero además, las condiciones sociales y culturales de la Europa del momento eran radicalmente diferentes a las de medio siglo antes, pues los pro- 
cesos de industrialización cambiaron drásticamente las normas de convivencia social imperantes hasta entonces. Los nuevos valores sociales que a finales del siglo XVIII habían inspirado la Revolución Francesa (el respeto hacia los otros, la libertad del individuo, la igualdad entre las personas, la solidaridad, etc.), habían calado hondo y se habían extendido como un reguero de pólvora por prácticamente todo el Continente gracias, en buena medida, a la cercanía humana que suponía el trabajo en espacios comunes y reducidos (las explotaciones mineras, las primeras industrias, etc.), tan diferentes a las antiguas y dispersas ocupaciones mayoritarias de la población, básicamente ligadas a la agricultura y la ganadería.

Los "nuevos" científicos no son inmunes a esa atmósfera de cambios sociales: la observación natural (al modo Hipocrático) y el pensamiento reflexivo (al modo filosófico) son sustituidos por la observación controlada y la necesidad de predecir de un modo fiable el curso de los acontecimientos. El escepticismo, el relativismo y el mecanicismo son las características dominantes de la "nueva ciencia". La posibilidad de experimentar con la realidad, o sea, de intervenir activamente en ella y no solo de observarla pasivamente, se convierte en la marca de contraste de la autentica actividad científica, contrapuesta a la reflexión filosófica. En consecuencia, los "nuevos" científicos necesitan involucrarse activamente en el mundo social en que viven, lo que a la larga tendrá una consecuencia inevitable: el científico individual (y no solo la ciencia en general) adquiere conciencia de su poder para influir en los cambios sociales. Esta es una de las razones que explica porqué el poder que hasta entonces ejercían las clases dominantes (vinculadas en gran parte a la religión) sobre la sociedad, va siendo inexorablemente sustituido por un nuevo poder, el de la ciencia y los científicos, mucho más capacitados que los antiguos poderes religiosos para dar respuestas inmediatas a las necesidades humanas.

Y es ésta atmósfera de cambios la que, según Reisman (1976) hace posible el surgimiento de nuevas ciencias: en Alemania la Psicología con Wilhelm Wundt, en Inglaterra la Antropología con James Prichard, y en Francia la Sociología con Auguste Comte.

La Psicología como campo de reflexión filosófica tenía una larga tradición, de la que podemos encontrar claros ejemplos en muchos de los grandes pensadores de todas las épocas y lugares: desde 
Avicena y Maimónides, pasando por Vives, Huarte de San Juan, Feijoó, Descartes, Spinoza, hasta llegar a Leibniz y Kant. Sin embargo, el interés de Wundt, que poseía una sólida formación filosófica, pero también biológica (era médico), era el de estudiar los contenidos conscientes de la mente humana y escogió las sensaciones como elementos o unidades básicas de la experiencia: no debe olvidarse que para Wundt, la psicología debía estudiar los fenómenos sensoriales accesibles a la experiencia inmediata consciente. Tanto la creación en Leipzig en 1879 del primer laboratorio de psicología, como el título de su primer libro Los Elementos de Psicología Fisiológica (1873-1874), responden a ese interés. Este libro, considerado por muchos como el primer texto de Psicología científica ${ }^{1}$ se constituyó en el vehículo de difusión de la nueva ciencia psicológica, con una decidida apuesta por vincularla al ámbito de la nueva orientación experimental de la ciencia, lo que la alejaría definitivamente de su antigua matriz filosófica.

Pero Wundt no es el único personaje al que debemos recurrir para reconstruir adecuadamente la historia de la psicología, ni Europa el único lugar que explica su origen y desarrollos. En esta historia Estados Unidos ha ejercido un papel nuclear que no deberíamos minimizar: por citar nombres concretos, William James no es un mero secundario en la historia de la psicología científica. Solo tres años después de la publicación del libro de Wundt, sale a la luz el texto de James Principios de Psicología (1890). Su opción por una "ciencia de la vida mental", que es como definía el objeto de la psicología, difería radicalmente de la propuesta de Wundt que, como no podía ser de otro modo, calificó el texto con las siguientes palabras "Es literatura, es bonito, pero no es psicología" (citado en Reisman, 1976, Pág. 22). También es verdad que James no ahorró elogios a la psicología experimentalista de Wundt, que calificaba como "psicología de los instrumentos de hojalata" y consideraba los experimentos como fríos, inútiles y aburridos, entre otras lindezas ("Como mucho (refiriéndose al método de Wundt), sirve para poner a prueba la paciencia y solo podría haber surgido en un lugar cuyos habitantes son proclives al aburrimiento...."). Su concepción de la vida mental en términos de

1. $Y$, en este contexto, científico es lo mismo que decir susceptible de someter sus postulados a los principios positivistas del control y la predicción, es decir, de la experimentación en su sentido más tradicional. 
cambio y flujo constante era desde luego poco compatible con la propuesta más atomizadora de los procesos mentales que defendía Wundt y, en consecuencia, los procedimientos (los métodos) para su abordaje diferían también de manera radical.

El anecdotario al respecto es extenso. Traerlo aquí a colación obedece únicamente a su interés como ejemplificación de un hecho²: desde su mismo nacimiento, la psicología ha estado presidida por disensos muy profundos que, como no podía ser de otro modo, han marcado su crecimiento y desarrollos posteriores tanto en lo conceptual como en lo aplicado o profesional. No obstante, hay que decir a continuación que tales disparidades y opciones contrapuestas no deberían ser amplificadas más allá de lo que en realidad son y representan: recuérdese que estamos asistiendo al nacimiento de una ciencia nueva que caminaba en busca de una identidad propia y diferenciada. $Y$ ya sabemos que la construcción de una identidad no es un proceso sencillo ni lineal.

¿Y qué decir de la historia de la psicología clínica? Si nos situamos en el contexto Europeo, es obligado recordar a Brentano quien, al mismo tiempo que Wundt publicaba sus Principios, hacía pública su Psicología desde el punto de vista empírico (1874). La noción de empírico se contrapone, en términos conceptuales, a la de experimento. Lo que pretendía recalcar Brentano es que los actos mentales, objetos de estudio propio de la psicología según él, debían ser netamente diferenciados de los fenómenos físicos, que sí constituían objetos susceptibles de experimentación. Por el contrario, el "sujeto psicológico", objeto de la psicología, era sin duda un hecho empírico constatable y real, pero no un objeto susceptible de experimentación. Los hechos psicológicos tenían intencionalidad, propósito y finalidad en sí mismos, y la única forma de abordarlos era mediante la intuición. Con estos planteamientos, Brentano acabaría por convertirse en el inspirador de dos de los modelos más influyentes en la historia del desarrollo de la psicología clínica europea: por un lado, la psicología de la Gestalt, inspiradora de diversos tipos de psicoterapia y

2. Como es obvio, el adecuado relato del devenir histórico de la psicología científica incluye otros muchos nombres, momentos históricos, y geografías que no serán mencionados ni tratados aquí. Nuestro interés no es reconstruir aquí la historia de la psicología (tarea que desborda la capacidad de la autora), sino simplemente recordar algunos datos y hechos históricos que ayuden a explicar (y a justificar) el hilo argumental que se intenta presentar. 
antecesora de algunos de los postulados actuales del modelo cognitivo; por otro, el movimiento fenomenológico liderado por Husserl, al que se vinculará posteriormente Karl Jaspers, "padre reconocido" de la Psicopatología.

De todos modos, y con la perspectiva que da el paso del tiempo, la influencia de Brentano sobre la futura psicología clínica a través de movimientos como los gestálticos o los fenomenológicos que se acaban de mencionar, fue más de orden conceptual (y más a largo plazo) que de tipo práctico (e inmediato). Porque, digámoslo ya desde el principio, si algo distingue el surgimiento de la psicología del de la psicología clínica, es la voluntad o el interés de aplicación inmediata de la segunda. Esto implica que su objetivo o finalidad última es buscar soluciones útiles para los problemas mentales de las personas. En este contexto, la descripción y el análisis pormenorizado de los procesos y estados mentales psicopatológicos al modo fenomenológico o gestaltista, requerían tiempo, paciencia, aptitudes de observación cuidadosa, y seguramente también sólidos conocimientos filosóficos, que no casaban demasiado con las "urgencias" aplicadas.

Por todo esto fueron mucho más importantes para la psicología clínica las influencias de Alfred Binet o Francis Galton, divulgadas y defendidas en Estados Unidos por James McKeen Cattell. El elemento común de estos tres grandes de nuestra historia era su interés por la medición de las características físicas y mentales de las personas y el reconocimiento del valor adaptativo de las diferencias individuales. Y sin duda, ese interés estaba mucho más cercano a la visión práctica-aplicada de la actividad científica que es la marca de contraste de la psicología clínica desde su inicio.

Con todos estos elementos no es de extrañar que el nacimiento "oficial" de la Psicología Clínica se produjera en Estados Unidos y no en Europa. Las razones de este "traslado geográfico" guardan relación con un aspecto muy característico de la cultura norteamericana de la época: cualquier actividad humana, incluyendo la científica, debe estar al servicio de la sociedad, y no al contrario. De aquí la conceptuación eminentemente práctica, aplicada, de la cultura y la actividad científicas.

Los Estados Unidos habían ido abandonando su dependencia ideológica de la vieja Europa y en las postrimerías del XIX era ya 
una nación grande, potente, y orgullosa de sí misma que hacía frente sin complejos y en todos los terrenos a las dos grandes potencias Europeas del momento, Alemania y Gran Bretaña. En ese proceso identitario tuvo mucho que ver, en opinión de Reisman (1976), la victoria rápida y sin paliativos que los norteamericanos obtuvieron en esa guerra que en España llamamos de Cuba (1898), pero que en realidad era una confrontación directa entre el caduco colonialismo europeo, representado en este caso por España, y la nueva visión del mundo y la sociedad que representaban los Estados Unidos. A que esa nueva visión se impusiera con tanta rapidez, ayudó sin duda el enorme crecimiento económico e industrial de los Estados Unidos en una época crucial de la industrialización en la que, además, no tuvo prácticamente competidores: recuérdese aquí la primera guerra mundial (1914-1918) que sumió a Europa en el desastre y la devastación más absolutos. Todo ello influyó decisivamente en que Estados Unidos se convirtiera en la primera potencia científica del mundo, lo cual es tanto como decir que prescribía cuáles eran los planteamientos y problemas que eran (o no eran) interesantes e importantes, en los que valía la pena invertir recursos y tiempo, y lógicamente también cuáles eran los métodos y procedimientos más útiles para su abordaje.

En el ámbito de la psicología, los planteamientos wundtianos que habían Ilegado a Estados Unidos en la versión solipsista e introspeccionista de Titchener, tenían pues poco futuro. Frente a ellos, reacciona el llamado "movimiento funcionalista americano" iniciado por William James, cuya una influencia en la psicología clínica sería desde luego determinante. En palabras de Korchin (1983, pág.5) "El enfoque funcionalista y pragmático de la psicología americana proporcionaba un terreno especialmente receptivo a la clínica y a otras psicologías aplicadas. El americano tenía poca paciencia para una psicología que diseccionaba hasta en sus más mínimos detalles las estructuras de la mente (...) así como para una psicología que especulaba en exceso sobre la naturaleza final de la mente humana".

En definitiva, es en este contexto en el que surge la psicología clínica, con una orientación aplicada de la psicología. Lo hace de la mano de un norteamericano, Lightner Witmer, que había estudiado con J. McK Cattell y que, siguiendo los pasos de éste, acudió también al laboratorio de Wundt en Leipzig (estancia que, según los historia- 
dores, aprovechó poco). Según Joseph Collins, Witmer describió la psicología clínica en su conferencia ante la American Psychological Association (APA) de 1896 en los siguientes términos: "La Psicología Clínica se deriva de los resultados obtenidos en el examen individualizado de muchos seres humanos (....) la clínica psicológica es una institución pública abierta al servicio de la sociedad, a la investigación propia, y a la formación de los estudiantes"

A Witmer le debemos pues la creación de esta modalidad profesional de la psicología, su denominación, y algunas de sus características distintivas. Pero poco más. Como dijeron Bernstein y Nietzel (1980, p. 44), "Witmer puso en marcha la Psicología Clínica, pero tuvo muy poco que ver con su desarrollo". Las causas de la escasa relevancia de Witmer en el desarrollo y la consolidación de la psicología clínica, hasta el punto de ser considerado por algunos como una mera anécdota histórica (Watson, 1953), son variadas y van desde su escaso interés por los desarrollos en el ámbito del diagnóstico de las capacidades mentales y la evaluación de las diferencias individuales, hasta su interés exclusivo en los problemas mentales infantiles. Pero a estas razones hay que añadir, según Reisman (1976) otra de índole político interno de la propia psicología: sus colegas de la APA no estaban preparados para concebir y aceptar una profesionalización de la Psicología, a la que seguían considerando más como una actividad científica "pura". Y menos aún que esa profesionalización se fuera a producir en el ámbito de la salud y la enfermedad mental. Todavía debían transcurrir más de 20 años para que se produjera el necesario cambio de mentalidad entre los psicólogos, de tal manera que fuera viable la inmersión de la psicología en el vasto y complejo mundo de las psicopatologías humanas. Y todavía unos años más para que el colectivo de psicólogos descubriera sus posibilidades profesionales en ese mundo.

El escenario que propició el cambio o la apertura hacia el mundo de la clínica psicológica entre los propios psicólogos fue, como es bien sabido, la Primera Guerra Mundial y, en especial, los años que van desde su finalización hasta el inicio de la segunda. De todos es conocido el impacto que esta guerra tuvo sobre el uso a gran escala de los tests, cuyos contenidos se ampliaron más allá de la evaluación de la inteligencia y que sirvieron para afianzar el rol del psicólogo como experto en la construcción, uso, e interpretación de estos ins- 
trumentos. Y ello además no solo, como hasta entonces, en el ámbito de la infancia, sino también en el de los adultos con problemas y trastornos mentales (Bernstein \& Nietzel, 1980). Se empezaron a desarrollar instrumentos estandarizados para evaluar la personalidad, los intereses o las emociones. Muchos de estos tests se basaban en los primeros planteamientos psicoanalíticos europeos, que habían llegado a los Estados Unidos gracias, entre otras cosas, a la invitación que G. Stanley Hall hizo a Freud para dictar una serie de conferencias en la celebración del 20 aniversario de la Clark University, en Massachusetts.

Pero es que, además, en esta época entre-guerras se produjo un declive vertiginoso de la psicología al modo wundtiano, propiciado tanto por el desmantelamiento económico e intelectual de las universidades centroeuropeas (no debe olvidarse que la Alemania nazi "abolió" en 1934 el psicoanálisis), como por el surgimiento de modelos y perspectivas acordes con la nueva psicología funcionalista dominante, que como ya se ha dicho, estaba mucho más interesada por las aplicaciones prácticas que por las teorías.

Por lo que hace a la psicología clínica, los modelos dominantes pasan a ser el psicoanálisis y el conductismo, que junto al desarrollo de los instrumentos de evaluación y medida de las diferencias individuales, proporcionan las primeras herramientas científicas, y "no biológicas" con las que los psicólogos clínicos pueden afrontar no solo el diagnóstico sino también el tratamiento de los trastornos mentales y del comportamiento.

\section{PSICOLOGÍA CLÍNICA: LOS OTROS ORÍGENES Y SUS CONSECUENCIAS}

Todo lo relatado hasta aquí refiere sin duda a los antecedentes de la psicología y de la psicología clínica, una de sus ramas profesionales más exitosas, y más o menos así se recogen en la mayoría de los textos autorizados y reconocidos. Pero estos antecedentes no son los únicos. Y a quien esto escribe, siempre le resultó difícil entender por qué (casi) nunca se hace referencia a esos "otros". En ocasiones, repasando los trabajos de los historiadores, se puede llegar a la conclusión de que hasta mediados del siglo XIX las relaciones 
entre psicología y psicopatología (entendida como marco conceptual de referencia de la psicología clínica) eran mínimas o inexistentes. Otras veces la impresión que queda es que la psicología clínica es un "invento" exclusivo de los psicólogos, que surge en el primer tercio del siglo $\mathrm{XX}$, y en el que no ha participado ningún otro colectivo científico ni profesional. $Y$ en cierto modo, lo mismo sucede con la psicología, aunque en este caso la amnesia histórica es menor ${ }^{3}$.

¿Cuáles son esos antecedentes de la psicología clínica diferentes a los estrictamente psicológicos? Básicamente pertenecen a dos aspectos íntimamente relacionados, por razones obvias: por un lado, los relativos a los conceptos y abordajes sobre los trastornos mentales; por otro, los de las personas concretas que los formularon y defendieron.

Que el interés por conocer las causas de los trastornos mentales y la búsqueda de procedimientos para su manejo es casi tan antiguo como la humanidad misma, lo sabe cualquiera. Que el modo de abordar su estudio científico ha sido predominantemente médicobiologista, también (al menos, desde el siglo XVII en adelante), lo cual no significa ni que los médicos que se dedicaban a ello tuvieran demasiado éxito, ni que la medicina otorgara un lugar relevante al estudio de las enfermedades mentales (sobre todo, en comparación con el interés prestado al resto de procesos mórbidos). Pero más allá de los éxitos o los fracasos, lo que resulta indiscutible es el hecho de que la medicina incluyó las psicopatologías humanas en el catálogo y en el terreno de las enfermedades, lo que entre otras cosas tuvo como consecuencia que los enfermos mentales fueran tratados como personas que requerían atención y cuidados específicos y especializados.

3. En la mayor parte de los textos de historia de la psicología se recogen los antecedentes de los siglos XVII, XVIII y principios del XIX en relación al futuro desarrollo de la psicología científica de finales del XIX: el interaccionismo psicofísico, la noción de automatismo, el empirismo psicológico, las distinciones entre las cualidades primarias y secundarias de las sensaciones, el asociacionismo, etc. Pero aún así, en ocasiones se tiene la impresión de que el surgimiento de la psicología es obra de un solo autor (Wundt, casi siempre), de una sola época (finales del XIX), o de un solo lugar (Alemania). 
El descontento y las limitaciones con los enfoques meramente biologistas de las psicopatologías por parte de los médicos que se ocupaban de estos enfermos, los psiquiatras pero también los neurólogos, hizo que muchos de ellos buscaran respuestas diferentes a las anatomopatológicas, fisiopatológicas, o hereditaristas de los siglos XVIII y XIX. Pero los psicólogos clínicos tendemos a olvidar (e incluso a obviar) que personas tan diferentes en opciones e influencias posteriores como Mesmer, Freud, Goldstein, Jaspers, Claparède, Rorschach, Pavlov, o McDougall eran médicos psiquiatras o neurólogos. Como médicos eran también Wundt y James. O en nuestro contexto más inmediato, Luis Simarro o Mira y López. Bien es verdad que la división de roles y las diferencias en preparación e intereses que existe actualmente entre ciencias y profesiones, no es comparable con lo que sucedía en la época de Wundt o en la de Freud, y que no podía haber psicólogos (ni psicólogos clínicos) sencillamente porque ninguna de estas ciencias y profesiones "existía" en sentido estricto. Pero este hecho no resta importancia al anterior: que en el origen de la psicología clínica (y en el de la psicología) se haya, también, una psiquiatría ( $\mathrm{y}$, en menor medida, una neurología) insatisfecha e incómoda con los planteamientos exclusivamente biomédicos sobre la enfermedad mental y su tratamiento 4 .

A medida que la psicología clínica fue desarrollándose y madurando como disciplina científica y adquiriendo a su vez estatus de profesión, empezaron a hacerse patentes los enfrentamientos y las disputas, en especial con los psiquiatras. Primero fueron los tests y sus aplicaciones para la evaluación y el diagnóstico, que fueron consideradas por muchos psiquiatras como un atentado y una amenaza a su autoridad hasta entonces indiscutible. $Y$ la verdad es que tenían razón: los psicólogos clínicos habían desarrollado (con ayuda muchas veces de los mismos psiquiatras) unos procedimientos nuevos y desde luego más útiles que los procedimientos diagnósticos

4. Como no puede ser de otro modo, también hay otras influencias históricamente relevantes para la psicología clínica, entre las que se encuentran desde luego la antropología cultural, la sociología, o la biología (especialmente la evolucionista). No obstante, en este trabajo se obviarán tales influencias con el fin de no distraer del argumento principal. 
típicos de la psiquiatría ${ }^{5}$ para la evaluación y el diagnóstico individual de las capacidades y problemas humanos. Un ejemplo de esta batalla perdida fue el requerimiento que en 1917 hizo la Sociedad de Psiquiatría de Nueva York a la American Psychological Association para prohibir el nombramiento de psicólogos como expertos en las comisiones oficiales encargadas de evaluar y diagnosticar los problemas mentales en la población escolar. El argumento era simple: estos problemas eran médicos, y por tanto solo los médicos estaban legitimados para evaluarlos, diagnosticarlos $\mathrm{y}$, por supuesto, tratar$\operatorname{los}^{6}$. Pero no tuvieron éxito, de modo que el efecto fue el opuesto al buscado: los psicólogos se encontraron con una legitimación formal que amparaba las funciones de evaluación y diagnóstico como una competencia propia.

La segunda amenaza para la profesión que fue denunciada por la psiquiatría oficial de la época fue la referida a la creciente implicación de los psicólogos clínicos en el tratamiento de los trastornos mentales. También en este caso tenían razón en percibir esa nueva competencia como una amenaza: los psicólogos clínicos estaban demostrando que eran capaces de diseñar y aplicar tratamientos eficaces para muchas de las afecciones y dolencias humanas. Eso era, sin duda, una amenaza ante unos tratamientos psiquiátricos costosos, en muchos casos dolorosos, con efectos secundarios indeseables, y con un grado de eficacia y utilidad más que dudoso. En 1954 la Asociación Médica Americana reivindicó la psicoterapia -entendida entonces como la práctica del psicoanálisis- como un procedimiento estrictamente médico. Y, por tanto, cualquier psicólogo que practicara psicoterapia, estaría incurriendo en una práctica ilegal y denunciable.

5. Me refiero a los interminables listados sobre enfermedades mentales y los infructuosos debates sobre la mejor forma de clasificarlas y describirlas que ocuparon el tiempo de los nosologistas del XIX y los primeros 70 años del XX.

6. Resulta cuanto menos curioso que en España, y con ocasión de la publicación en 1998 del Real Decreto que institucionalizó la psicología clínica como una profesión sanitaria propia de los psicólogos, varias organizaciones de la Psiquiatría del país (y la Organización Médica Colegial en bloque) denunciara las funciones que en ese Decreto se atribuían como propias (aunque no exclusivas, claro está) a los psicólogos clínicos: evaluación, diagnóstico y tratamiento de los trastornos mentales y del comportamiento. La argumentación de base era prácticamente la misma que la enunciada de 1917 por la Sociedad de Psiquiatría de NuevaYork: esas funciones eran competencias médicas, y solo los médicos podían realizarlas. Tampoco aquí tuvieron éxito con la denuncia. 
También esta batalla la perdieron los psiquiatras, tanto a medio como a largo plazo. Como muestra de que esta no es una afirmación gratuita ni triunfalista, se remite al lector a las principales bases de datos internacionales e institutos de investigación de buenas prácticas en el ámbito de la salud y la enfermedad humanas (un ejemplo, pero no el único, es el National Institute for Clinical Excellence, NICE, británico). En lo tocante a las enfermedades y trastornos mentales y del comportamiento, los tratamientos eficaces que se recomiendan hoy día como abordaje imprescindible y de primera línea, son los psicológicos en el caso de los trastornos más prevalentes, sea cual sea su gravedad, dejándose los psiquiátricos (restringidos a la farmacoterapia casi siempre) para un segundo o tercer nivel y como co-adyuvantes de los tratamientos psicológicos sensu stricto. Como es natural, no todos los tratamientos psicológicos actualmente disponibles gozan del mismo reconocimiento, pues son los tratamientos cognitivo-conductuales los que, en su inmensa mayoría, se recomiendan y avalan como eficaces y eficientes. Pero lo que resulta indudable es que estos tratamientos son inequívocamente propios de la psicología clínica, en el sentido de que han sido desarrollados y puestos a prueba por psicólogos clínicos. La pregunta que surge de inmediato aquí es por qué la psicología clínica, en comparación con la psiquiatría, ha sido capaz de desarrollar tratamientos eficaces y útiles para una buena parte de las psicopatologías humanas. Seguramente hay más de una buena respuesta a este interrogante, y se nos ocurren algunas de ellas. Pero este trabajo trata sobre la psicología clínica y no sobre la psiquiatría, de manera que no parece procedente abundar en este tema. Preguntémonos, pues, por las razones del éxito de la psicología clínica.

\section{ALGUNAS RAZONES DEL ÉXITO DE LA PSICOLOGÍA CLÍNICA.}

El enfoque psicodinámico de los trastornos mentales, iniciado en Francia por Mesmer y desarrollado mucho después por Braid en Inglaterra y por Liebault, Bernheim y Charcot $^{7}$ en Francia, enfatizó el poder que tienen las experiencias inconscientes en el desarrollo de 
las personas, y proporcionó a la psicología clínica no solo una nueva concepción de la enfermedad mental, según la cual ésta podía tener, al menos en parte, una naturaleza psicológica, sino además un nuevo modo de tratamiento, basado en dos grandes pilares: la fuerza de la palabra y la implicación del propio sujeto en su proceso de curación. El nuevo modelo abrió las puertas a la posibilidad de desarrollar tratamientos psicológicos, opuestos a los tratamientos médico-físicos que eran los únicos disponibles hasta entonces.

Ahora bien: no se olvide que el modelo psicodinámico había surgido en el ámbito médico y, entre otras cosas, permitía ampliar el rango de intervenciones terapéuticas, extraordinariamente limitadas para los psiquiatras por ese entonces. Desde ahí cabe entender la prohibición decretada por la Asociación Médica Americana, a la que antes se hizo referencia, de que los psicólogos clínicos dispensaran psicoterapia (es decir, psicoanálisis). Es posible que esta prohibición alentara a los psicólogos clínicos a desarrollar otros modos de psicoterapia alternativos al psicoanálisis. $Y$ bien entrados los años cincuenta, estos modos comienzan a hacerse bien visibles: desde las terapias breves (Rogers, Ellis, etc.), hasta la terapia y modificación de conducta, sólidamente asentada en el conductismo. Y con el conductismo comienza verdaderamente el despegue científico y profesional de la psicología clínica.

El conductismo es el primer gran modelo psicológico que abraza sin dudarlo los principios teóricos y metodológicos del programa positivista imperante en la ciencia. Era, además, un modelo fácilmente "transportable" al contexto clínico: permitía explicar, diagnosticar y tratar cualquier trastorno mental, sin exclusión, fuera cual fuese la edad en que éste se presentara, de forma individualizada o en grupo, dependiendo de la naturaleza del problema y de los objetivos del tratamiento. Y sobre todo permitía hacer todo eso desde dentro de la Psicología, lo que evitaba enredarse en estériles disputas "territoriales" y o conceptuales con los psiquiatras.

Con el transcurso del tiempo, el enfoque conductista radical se irá complementando con otras opciones y modelos teóricos, de los que quizá el más importante sea el cognitivo, tanto en su versión más dura y experimentalista de los años 70 y 80 del pasado siglo (procesamiento de información), como en otra más amplia y compre- 
hensiva en la que se incluyen los enfoques y procedimientos de la investigación social o los de la personalidad. El ámbito de la psicología clínica en el que más impacto ha tenido la adopción del modelo cognitivo es en el de la investigación psicopatológica y en el subsiguiente desarrollo de procedimientos de tratamiento psicológico (las terapias cognitivas). De hecho, la psicopatología y la terapia cognitivas han sido probablemente dos de las áreas de investigación psicológica que más han progresado en los últimos 30 años, generando un número importante de modelos explicativos de trastornos mentales complejos sobre los que el conductismo no pudo aportar prácticamente nada, tales como la esquizofrenia, los trastornos del estado de ánimo, los trastornos graves de ansiedad, las somatizaciones, los trastornos de personalidad, o los trastornos del comportamiento alimentario, para los que se han propuesto, además, tratamientos específicos y como antes se mencionó, de probada y reconocida utilidad.

Pero más allá de los modelos o escuelas de la psicología clínica y sus aportaciones específicas, todos ellos comparten una misma perspectiva acerca de qué cabe considerar como trastorno mental humano y, por tanto, como objeto lícito de conocimiento para la psicología clínica: la relatividad socio-cultural de ese objeto. Esto es, el reconocimiento de que las ideas sobre la salud mental que mantiene una sociedad son determinantes para la construcción de las ideas sobre su carencia o ausencia.

Así pues, el objeto de estudio e interés propio de la psicología clínica va más allá de la idea de "enfermedad mental" para incluir todas las experiencias, sentimientos, y/o actividades, mentales o comportamentales de las personas que resultan inusuales o anómalas (en el sentido que lo formuló Reed, 1988), disfuncionales y dañinas (tal y como lo ha definido Wakefield, 1992, 1997), inadaptadas y fuera del control o la voluntad personales (según el concepto de Widiger y Trull, 1991; Widiger y Sankis, 2000), o que provocan interferencias o deterioro en el desarrollo personal, en el comportamiento, y en las relaciones sociales. Todos estos aspectos, como se ha dicho, van más allá de los límites estrictos que, en términos conceptuales, impone la noción de "enfermedad" cuando se aplica a las psicopatologías humanas. 
Junto a estos postulados comunes, la mayoría de los enfoques o escuelas de la psicología clínica actual comparte asimismo el supuesto de que las experiencias, sentimientos y actividades mentales o comportamentales se distribuyen a lo largo de varias dimensiones, lo que implica que es necesario considerar en qué grado se presentan en un momento dado y a lo largo del tiempo (estabilidad), qué variables median en su incremento y en su atenuación, y en qué grado difieren de la normalidad. Por último, en el plano metodológico, se comparte la noción de que la investigación de las psicopatologías puede llevarse a cabo tanto en situaciones naturales como en condiciones artificiales (vgr. de laboratorio o experimentales). En el segundo caso, es necesario que se reproduzcan con la máxima fidelidad posible los contextos en los que se produce de forma natural el fenómeno a estudiar. Se consideran como fuentes de datos útiles tanto los procedentes de la información subjetiva --desde los informes introspectivos, las historias de vida, o los relatos autobiográficos, hasta los cuestionarios o instrumentos estandarizados--, como los directamente observables desde "fuera" del individuo --incluyendo en este caso no solo parámetros tales como la latencia de respuesta, el comportamiento motor, o el rendimiento en una tarea, sino también las informaciones y relatos que observadores cercanos al individuo puedan aportar sobre su malestar o su sufrimiento, ya sea éste propio o causado a terceros.

Pese a lo expuesto, sería erróneo pensar que existe un planteamiento unitario o unánime sobre la psicología clínica. Entre otras cosas, porque tampoco hay planteamientos unitarios sobre lo que se entiende hoy por "psicología clínica". Esta rama de la psicología ha ido ampliando progresivamente sus marcos de referencia hasta tal punto que bajo el apellido "clínico" es posible hoy encontrar modelos y explicaciones tan diferentes como los derivados de la inteligencia artificial y el procesamiento de información, los neuropsicológicos, los conductuales-cognitivos, los sociales-constructivistas, y otros muchos. No obstante lo dicho, la influencia del enfoque cognitivoconductual destaca en gran medida de los demás, hasta el punto de configurarse hoy como el paradigma o modelo dominante y el que disfruta de un mayor éxito científico (y también social), tal y como queda patente en los repertorios de "tratamientos eficaces" a los que se hacía referencia en el apartado anterior. 
Por lo que se refiere al diagnóstico de los trastornos mentales, el papel de la psicología clínica es menos relevante que el logrado en el ámbito de la investigación psicopatológica y en el de las psicoterapias, a pesar de que los primeros psicólogos clínicos eran, antes que nada, expertos en evaluación. El triunfo indiscutible de las nosologías psiquiátricas sobre las alternativas diagnósticas de la psicología clínica (reducidas poco menos que al análisis funcional), ha tenido y está teniendo efectos nocivos sobre el desarrollo de procedimientos de evaluación y diagnóstico alternativos o diferentes a los que "dictan" los "DSMs" y las "CIEs". Y lo peor es que, al menos a corto plazo, no se vislumbra una solución factible para la psicología clínica, por mucho que psicólogos clínicos de prestigio formen parte de los comités encargados de elaborar los criterios diagnósticos de los trastornos mentales, tanto en el caso de la American Psychiatric Association, como en el de la Organización Mundial de la Salud.

\section{LA CONTRIBUCIÓN ACTUAL DE LA PSICOLOGÍA AL ÉXITO DE LA PSICOLOGÍA CLÍNICA}

¿En qué ha contribuido la psicología a estos éxitos de la psicología clínica?. La respuesta políticamente correcta sería que la psicología clínica no existiría sin la psicología, puesto que aquella es una rama o extensión profesional-aplicada de ésta. Y eso es tanto como reconocer que la psicología es la fuente de la que provienen las buenas preguntas, los datos, los métodos, los instrumentos, y hasta los resultados que después los psicólogos clínicos, en un alarde de eficacia, creatividad, e inteligencia práctica, aplican sin cuestionamientos importantes a la evaluación, el diagnóstico y el tratamiento de los trastornos mentales y del comportamiento. Pero la verdad es que las cosas son bastante diferentes, en especial si nos referimos a los últimos 25 años.

En primer lugar, porque la psicología, tal y como podíamos entenderla hace 50 años, no existe hoy. Lo que existen son psicólogos (y psicologías) que investigan en, y se interesan por, la memoria, o las emociones, o las redes sociales, o las etapas evolutivas, o el pensamiento, o la personalidad, por poner algunos ejemplos. Y las relaciones entre unos y otros son escasas, cuando no claramente inexistentes. 
En segundo término, porque también entre los psicólogos clínicos existe no solo diversidad de orientaciones teóricas y metodológicas, sino también de intereses y motivaciones: el psicólogo clínico que se dedica a investigar y tratar los trastornos emocionales, tiene ante sí una tarea bastante diferente a la del que se ocupa de las drogadicciones, o del que se centra en la rehabilitación de los trastornos mentales y neurológicos, o el que se interesa por los procesos de enfermar no mentales (vgr., psicología clínica de la salud).

Esta atomización de intereses y obligaciones revela, sin duda, la madurez de una ciencia y una profesión que hace poco más de 100 años daban sus primeros pasos reconocidos. Por tanto, no parece que haya que ponerse dramáticos ante la situación actual, ni tiene sentido añorar tiempos pasados, entre otras cosas porque ahora estamos bastante mejor que cuando empezamos. Pero al mismo tiempo, es importante ser conscientes de esta dispersión, porque nos va en ello la identidad. Lo que identifica a los psicólogos (del pensamiento, de la motivación, o de cualquier otro ámbito "básico") y a los psicólogos clínicos (en tanto que profesionales aplicados de la psicología) como miembros de un mismo colectivo científico-yprofesional, no es tanto sus intereses del momento, como su actitud ante los problemas a resolver, lo que es tanto como decir marco epistemológico de referencia, preparación teórica de base, métodos, y preparación para el ejercicio profesional. También nos identifica (o debería hacerlo), aquello que NO somos: no somos médicos, ni enfermeros, ni sociólogos, ni genetistas, ni pedagogos, ni antropólogos, ni .....

Por tanto, adoptar intereses, actitudes, conceptos, y metodologías que son más propias de la medicina, la enfermería, la genética, la sociología, etc.., a expensas de (o sea, en sustitución de) los propiamente psicológicos, solo puede suponer una pérdida de identidad y de posibilidades de crecimiento y desarrollo colectivos. La mayoría de psicólogos clínicos conoce bien este riesgo, y suele estar bien atento a sus peligros. Pero los hay también que, ya sea por inseguridad, por desconocimiento, o por presiones sociales y profesionales, dudan de su papel, de su estatus y, lo que es peor, de sus posibilidades científicas y profesionales. 
También es verdad que resulta a veces difícil buscar (y encontrar) en la psicología no-clínica datos y procederes útiles para nuestros fines como psicólogos clínicos. Al mismo tiempo, es más sencillo recurrir a aquello que tenemos más cercano, la psiquiatría, con la que compartimos no solo espacios físicos, sino también problemas y urgencias que resolver. Esto es especialmente notable en el caso del diagnóstico, pero también en el excesivo recurso a los tratamientos farmacológicos que en muchos casos son alentados y recomendados por los propios psicólogos clínicos, como si tales tratamientos fueran realmente los de elección.

Pero también hay otras razones que no conviene pasar por alto: en muchas más ocasiones de lo deseable, la psicología clínica no puede hacer uso de los recursos psicológicos porque o bien no tiene garantías de que le permitan resolver el problema que tiene delante $(\mathrm{y}$ que necesita solución), o bien porque la solución que aportan es poco viable. Pongamos un ejemplo típico: el uso de instrumentos estandarizados para la evaluación y el diagnóstico. El psicólogo clínico no puede pedir a un "cliente" que acude a solicitar ayuda por, digamos, una fobia social o una dependencia alcohólica, que responda a una lista interminable de preguntas, no siempre bien redactadas o cercanas al lenguaje cotidiano, con el fin de averiguar cuáles los aspectos positivos y negativos de su personalidad, o su forma de recibir y expresar emociones y afectos, o su mayor o menor predisposición a reaccionar con miedo excesivo ante diversas situaciones. $Y$ no puede hacerlo no solo por razones de ética o urgencia, sino también porque no le son útiles, porque no le dicen nada que no pueda averiguar con unas pocas preguntas bien orientadas, o porque sencillamente no están ni pensadas ni formuladas para el ámbito clínico.

En lo tocante a la investigación, la situación no es muy diferente: al psicólogo clínico le resulta extraordinariamente difícil, cuando no imposible, obtener siquiera ideas de muchos ámbitos de la investigación psicológica no clínica que pueda desarrollar y aplicar para mejorar su comprensión de los problemas a los que se enfrenta en su trabajo diario. De ahí que, en muchas ocasiones, sean los propios psicólogos clínicos los que desarrollan sus propios programas de investigación sin tener en cuenta (ni tampoco preocuparse por ello) los que se realizan en otros ámbitos de la psicología no clínica. 
Esta situación de incomunicación entre psicólogos no-clínicos y psicólogos clínicos lleva en muchas ocasiones a afirmaciones del tipo siguiente por parte de los primeros: "a los psicólogos clínicos no les interesa la investigación" o "los psicólogos clínicos no saben psicología", o "lo único que les interesa a los psicólogos clínicos es que les den una buena receta y, si es breve, mejor". Por su parte, los psicólogos clínicos se alejan progresivamente del resto del colectivo de psicólogos, de quienes se quejan de no ser atendidos ni escuchados, o simplemente de que aquellos "no le aportan nada nuevo". $Y$ así, ignorantes los unos de los otros, el abismo se hace cada vez más insalvable y las posibilidades de enriquecimiento mutuo (y de preservación de una identidad común) se van desvaneciendo.

\section{RIESGOS DEL ÉXITO: COMPETENCIAS E INCOMPETENCIAS DE LA PSICOLOGÍA CLÍNICA}

Antes hemos aludido a la diversidad de problemas a los que se enfrenta la psicología clínica y a las presiones y urgencias de una sociedad que exige respuestas rápidas y eficaces. Añadamos ahora la diversidad de enfoques, contextos, y métodos que pueblan el quehacer del clínico, y tendremos los dos elementos principales del cóctel explosivo en el que se encuentra actualmente la psicología clínica. En un trabajo anterior (Belloch, 2005) aludía también a este problema real y, a día de hoy, sigo pensando lo mismo: el argumento de que el psicólogo clínico aplicado debe ser capaz de resolver cualquier problema de salud y enfermedad mental que se le plantee, independientemente de cuál sea el nivel de sus conocimientos como científico, o los de la psicología clínica misma, esconde simple y llanamente una temeraria carencia de autocrítica y auto-reconocimiento de los propios límites (y de los límites de la profesión). No se puede ofrecer como legítimo, algo que no lo es. Y una práctica de la psicología clínica que no esté científicamente fundamentada, no solo no es legítima, sino que además puede añadir más daño al problema que se pretende solventar.

Cualquier actividad profesional que desarrolle un psicólogo clínico debe estar firmemente anclada en los conocimientos científicos disponibles hasta ese momento, y por tanto, es responsabilidad de cualquier clínico asegurarse de que aquello que prescribe y realiza 
en la práctica, es científicamente válido y aceptable. De no hacerlo así, su práctica debería considerarse ilegítima, y en lugar de hablar de ciencia psicológica estaríamos ante una pseudociencia. En palabras de McFall (1991) "We simply cannot afford to purchase superficial tranquility at the expense of integrity".

El dilema de la "urgencia" en ofrecer una respuesta, es simplemente tramposo. Ante la urgencia caben dos posibles respuestas, igualmente válidas: la primera, reconocer que no se sabe cómo responder, que como psicólogo clínico no se dispone todavía de una buena respuesta para el problema que, por ejemplo, nos plantea un paciente, un familiar, o un colega; y la segunda, que la respuesta, la solución, o el remedio que se pueden proporcionar no está todavía suficientemente probada en términos de su validez y utilidad reales, es decir, lo que toda la vida hemos entendido como "en fase de estudio o en fase de investigación". Es decir, estamos obligados a que el destinatario de nuestra actividad sepa con claridad meridiana, que lo que por ahora le podemos ofrecer no está exento de riesgos, que no sabemos todavía cuál es su utilidad real, porque se haya todavía en fase de estudio y validación. Esto que es tan obvio, que tan legítimamente reclamamos como ciudadanos ante cualquier producto, y más si ese producto tiene que ver con nuestra salud, no es algo que siempre esté presente en nuestra actividad cotidiana como psicólogos clínicos.

En definitiva: la psicología clínica como cualquier otra actividad científica-y-profesional no es competente para todo ni para todos. $Y$ reconocer que todavía somos incompetentes para muchas cosas y en muchos ámbitos, no es ningún descrédito. La sensación de omnipotencia que muchas veces se constata por lo que se refiere a las posibilidades de actuación científica y profesional de los psicólogos clínicos, llegan hasta el punto de parecer que todos servimos para todo, que somos capaces de todo, y que podemos solventar cualquier problema de salud (mental o no mental) que se nos ponga por delante: desde el tratamiento de una enuresis infantil hasta la rehabilitación de una toxicomanía, pasando por la resolución de un problema de pareja, el tratamiento de una crisis de ansiedad, o la reintegración a la normalidad de las funciones cognitivas superiores en un accidente cerebro-vascular. Esta actitud acrítica sobre lo que podemos y sabemos es enormemente perjudicial y peligrosa, no 
solo para los psicólogos clínicos en tanto que colectivo profesional, sino sobre todo para nuestros clientes.

También es verdad que la indefinición de competencias y capacidades afecta no solo a los psicólogos clínicos sino también a los distintos ámbitos profesionales en los que se desenvuelven los psicólogos. Así, no es infrecuente encontrarse con debates sobre si un psicólogo educativo puede proporcionar un tratamiento psicológico específico para tratar un trastorno mental que presenta un escolar, o si un psicólogo dedicado a la selección de personal está capacitado para hacer diagnósticos y tratamientos relacionados con la salud mental de los trabajadores de una empresa. No voy a entrar aquí en semejante cuestión, pero no me resisto a apuntarlo como indicador de la indefinición de roles y capacidades en la que nos estamos moviendo en muchas ocasiones. Es evidente que todo psicólogo está capacitado por ley (como lo está cualquier profesional recién licenciado en España) para realizar cualquier actividad relacionada con su ámbito profesional: desde una selección de personal, hasta aplicar un tratamiento psicológico específico para un trastorno delirante, o un programa de rehabilitación de un esquizofrénico crónico, o un programa de counseling, y así hasta el infinito. La pregunta es si semejante "amplitud" de capacidades es posible y éticamente sostenible.

Por resumir: el excesivo énfasis que en no pocos momentos se pone en defender que un psicólogo es competente para actuar en cualquier ámbito y para cualquier problema de salud, independientemente de si ha recibido o no preparación específica para ello, no solo revela un desconocimiento atroz (y una peligrosa incompetencia) por parte de quienes tan alegremente defienden tales "derechos", sino que además sitúa a la psicología clínica (y a la propia psicología) al borde la extinción como actividad científica de prestigio. Y lo que es mucho peor, juega despiadadamente con las esperanzas de muchas personas y pone en peligro su salud mental. 
Otra vertiente del debate sobre las competencias de los psicólogos y las de los psicólogos clínicos que ha sido recientemente objeto de debates y discusiones enfrentadas en España, es la confusión entre lo que significa una actividad clínica y una sanitaria (en ambos casos, con referencia a la psicología, claro está). Así, en los escritos, noticias, debates, etc. aun vigentes, tiende a confundirse lo que es en sentido estricto una actividad clínica con lo que resulta una actuación sanitaria, dirigida a mejorar o preservar la salud de los ciudadanos. Se apela, por ejemplo, a la definición de la OMS para delimitar el ámbito de "lo sanitario", lo que sin duda es un argumento de autoridad, para después dar un salto conceptual que confunde la definición de salud que ese organismo promueve, con lo que significa profesión o actividad sanitaria, asunto sobre el cual la OMS no se pronuncia en absoluto, que se sepa. Tampoco se pronuncia este organismo sobre quién o quiénes tienen las competencias para cuidar de la salud de las personas, ni desde el punto de vista de las administraciones concretas (qué Ministerios, o qué entidades concretas de la Administración de un Estado, por ejemplo), ni desde el de los profesionales especialmente competentes y capacitados para tal misión.

Otro de los argumentos que está presente en este debate hace referencia a la necesidad de considerar la salud (y el cuidado de la salud por los profesionales) desde un modelo bio-psico-social, que tenga en cuenta no solo a la persona en su unidad psico-física, sino también al contexto social en el que vive, los roles que desempeña, etcétera. $Y$ se dice que el psicólogo sigue ese modelo en su actividad profesional, porque tiene esa formación y está especialmente preparado para ello. A partir de ahí, se afirma que el psicólogo es un profesional sanitario, porque en cualquier ámbito de desempeño profesional su objetivo último es cuidar de, o mejorar, la salud de las personas, las instituciones o los grupos. Todo esto es cierto y no se pone en cuestión.

Lo cuestionable surge cuando se confunde "objetivo último" con "objetivos concretos, medios y capacitación para lograrlos, y ámbito de actuación". Dicho de otro modo, tal y como se están manejando los argumentos, toda actividad que desarrolle una persona (psicólogo o no) está dirigida a mejorar la salud propia o ajena (exceptuando a los criminales, y a algunas psicopatologías, claro). Pero ello no significa que el concepto de lo sanitario sea el más adecuado para definir una 
actividad profesional concreta. Además, este modo de argumentar olvida o contradice el supuesto del que parte (el modelo bio-psicosocial), pues parecería según tales argumentos, que la actividad de un psicólogo de empresa, es indistinguible de la de un psicólogo educativo, un psicólogo clínico, o un psicólogo de la actividad física y el deporte. Es decir: la reducción de los cuidados de salud al ámbito de lo sanitario contraviene el enfoque bio-psico-social, que precisamente amplía el concepto de cuidados de salud desde un enfoque biologista estricto y decimonónico, a la inclusión de los aspectos psicosociales involucrados (por ejemplo, el ámbito en el que desempeña su actividad un psicólogo que evalúa y diagnostica el grado de minusvalía de una persona, o su adecuación a un puesto laboral concreto, o el ámbito educativo en el que un psicólogo valora las dificultades de aprendizaje de un estudiante y promueve estrategias para su mejora), además de a la consideración de lo psicológico-intrapsíquico (la percepción y valoración que una persona hace de su estado de salud, el modo en que experimenta o vive su enfermedad, los procesos mentales involucrados en la génesis y en el mantenimiento de los trastornos mentales, sus características básicas de personalidad, y un largo etc.).

Con todo, más extraño resulta la asimilación de lo sanitario a lo clínico: la evaluación, el diagnóstico y la intervención y tratamiento psicológicos de un problema, un individuo, o un grupo, difieren en función de cuáles sean sus objetivos y fines, y el ámbito en que se produzcan. No se requiere el mismo tipo de preparación para hacer un diagnóstico de minusvalía, o para evaluar la aptitud de una persona para conducir un vehículo, que para realizar (o descartar) un diagnóstico de depresión o de estrés post-traumático y planificar, llevar a cabo, y evaluar, el tratamiento psicológico más adecuado. Es decir: la amplitud y diversidad de roles y ámbitos de desempeño profesional de los psicólogos va más allá de lo clínico y de lo sanitario.

Dicho en otros términos: del mismo modo que no toda actividad profesional de los psicólogos es reductible ni asimilable a lo sanitario, no toda actividad psicológica sanitaria es asimilable a actividad clínica. De lo contrario, ¿qué sentido tendría entonces distinguir entre distintos perfiles profesionales de los psicólogos y plantear, además, requisitos y necesidades diferentes de formación, tal y como plantean y defienden con razón las organizaciones científicas y profesionales de la psicología en casi todo el mundo? 
Para terminar: es evidente que queda mucho camino por recorrer y mucho que afianzar y demostrar. El viejo dicho "quien mucho abarca, poco aprieta" resume bien lo dicho hasta aquí. Pero sin olvidar lo que dejó dicho el poeta "caminante no hay camino, se hace camino al andar". Sigamos pues caminando.

\section{REFERENCIAS}

Belloch, A. (1997). One hundred years of clinical psychology: roots, doubts and hopes. En R.Fuller, P.Noonan Walsh y P.McGinley (Eds) A Century of Psychology. Londres: Routledge. Págs. 85-106.

Belloch, A. (2005). ¿Qué es un psicólogo clínico?: En torno a la preparación, competencias y desarrollos de una profesión. Análisis y Modificación de Conducta, 31, 235-272.

Bernstein, D.A. y Nietzel, M.T. (1980). Introduction to Clinical Psychology. Nueva York, McGraw Hill.

Korchin, S.I. (1983). The history of clinical psychology: a personal view. En M. Hersen, A.E. Kazdin y A.S. Bellack (eds.). The Clinical Psychology Handbook (p. 5-19). Nueva York, Pergamon Press.

McFall, R.M. (1991). Manifesto for a Science of Clinical Psychology. The Clinical Psychologist, 44, 75-88.

Reed, G. (1988). La psicología de la experiencia anómala. Valencia, Promolibro.

Reisman, I.M. (1976). A History of Clinical Psychology. Nueva York, Irvington.

Wakefield, J.C. (1992). The concept of mental disorder: on the boundary between biological facts and social values. American Psychologist, 47, 373-388.

Wakefield, J.C. (1997). Diagnosing DSM-IV-Part I: DSM-IV and the concept of disorder. Behavior Research and Therapy, 35, 633-649.

Widiger, T.A., y Sankis, L.M. (2000). Adult Psychopathology: Issues and controversies. Annual Review of Psychology, 51, 377-404.

Widiger, T.A., y Trull, T.J. (1991). Diagnosis and clinical assessment. Annual Review of Psychology, 42, 109-133 
